\title{
Stochastic Delay Population Dynamics under Regime Switching: Permanence and Asymptotic Estimation
}

\author{
Zheng Wu, Hao Huang, and Lianglong Wang \\ School of Mathematical Sciences, Anhui University, Hefei 230039, China \\ Correspondence should be addressed to Lianglong Wang; wangll@ahu.edu.cn
}

Received 30 January 2013; Accepted 14 March 2013

Academic Editor: Yuming Chen

Copyright ( 2013 Zheng Wu et al. This is an open access article distributed under the Creative Commons Attribution License, which permits unrestricted use, distribution, and reproduction in any medium, provided the original work is properly cited.

\begin{abstract}
This paper is concerned with a delay Lotka-Volterra model under regime switching diffusion in random environment. Permanence and asymptotic estimations of solutions are investigated by virtue of $V$-function technique, $M$-matrix method, and Chebyshev's inequality. Finally, an example is given to illustrate the main results.
\end{abstract}

\section{Introduction}

The delay differential equation

$$
\frac{d x(t)}{d t}=x(t)(a-b x(t)+c x(t-\tau))
$$

has been used to model the population growth of certain species and is known as the delay Lotka-Volterra model or the delay logistic equation. The delay Lotka-Volterra model for $n$ interacting species is described by the $n$-dimensional delay differential equation

$$
\frac{d x(t)}{d t}=\operatorname{diag}\left(x_{1}(t), \ldots, x_{n}(t)\right)(b+A x(t)+B x(t-\tau)),
$$

where $x=\left(x_{1}, \ldots, x_{n}\right)^{T} \in R^{n}, b=\left(b_{1}, \ldots, b_{n}\right)^{T} \in R_{+}^{n}, A=$ $\left(a_{i j}\right)_{n \times n} \in R^{n \times n}, B=\left(b_{i j}\right)_{n \times n} \in R^{n \times n}$. There is an extensive literature concerned with the dynamics of this delay model and have had lots of nice results and we here only mention the work of Ahmad and Rao [1], Bereketoglu and Györi [2], and Freedman and Ruan [3], and in particular, the books by Gopalsamy [4], Kolmanovskiǐ and Myshkis [5], and Kuang [6] among many others.

In the equations above, the state $x(t)$ denotes the population sizes of the species. Naturally, we focus on the positive solutions and also require the solutions not to explode at a finite time. To guarantee the positive solutions without explosion (i.e., the global positive solutions), some conditions are in general needed to impose on the system parameters. For example, it is generally assumed that $a>0, b>0$, and $c<b$ for (1) while much more complicated conditions are required on matrices $A$ and $B$ for (2) [7] (and the references cited therein).

On the other hand, population systems are often subject to environmental noise, and the system will change significantly, which may change the dynamics behavior of solutions significantly $[8,9]$. It is therefore necessary to reveal how the noise affects the dynamics of solutions for the delay population systems. In fact, many authors have discussed population systems subject to white noise [7-18]. Recall that the parameter $b_{i}$ in (2) represents the intrinsic growth rate of species $i$. In practice, we usually estimate it by an average value plus an error term. According to the well-known central limit theorem, the error term follows a normal distribution. In terms of mathematics, we can therefore replace the rate $b_{i}$ by $b_{i}+\sigma_{i} \dot{w}(t)$, where $\dot{w}(t)$ is a white noise (i.e., $w(t)$ is a Brownian motion) and $\sigma_{i} \geq 0$ represents the intensity of noise. As a result, (2) becomes a stochastic differential equation (SDE, in short)

$$
\begin{aligned}
d x(t)= & \operatorname{diag}\left(x_{1}(t), \ldots, x_{n}(t)\right) \\
& \times[(b+A x(t)+B x(t-\tau)) d t+\sigma d w(t)],
\end{aligned}
$$

where $\sigma=\left(\sigma_{1}, \ldots, \sigma_{n}\right)^{T}$. We refer to [7] for more details. 
To our knowledge, much attention to environmental noise is focused on white noise. But another type of environmental noise, namely, color noise, say telegraph noise, has been studied by many authors (see [19-25] and the references cited therein). In this context, telegraph noise can be described as a random switching between two or more environmental regimes, which differ in terms of factors such as nutrition or rain falls $[23,24]$. Usually, the switching between different environments is memoryless and the waiting time for the next switch has an exponential distribution. This indicates that we may model the random environments and other random factors in the system by a continuoustime Markov chain $r(t), t \geq 0$ with a finite state space $S=\{1,2, \ldots, N\}$. Therefore, stochastic delay population system (3) in random environments can be described by the following stochastic model with regime switching:

$$
\begin{aligned}
& d x(t)= \operatorname{diag}\left(x_{1}(t), \ldots, x_{n}(t)\right) \\
& \times[(b(r(t))+A(r(t)) x(t)+B(r(t)) x(t-\tau)) d t \\
&\quad+\sigma(r(t)) d w(t)] .
\end{aligned}
$$

The mechanism of ecosystem described by (4) can be explained as follows. Assume that, initially, the Markov chain $r(0)=\iota \in S$. Then the ecosystem (4) obeys the SDE

$$
\begin{aligned}
& d x(t) \\
& =\operatorname{diag}\left(x_{1}(t), \ldots, x_{n}(t)\right) \\
& \quad \times[(b(\iota)+A(\iota) x(t)+B(\iota) x(t-\tau)) d t+\sigma(\iota) d w(t)],
\end{aligned}
$$

until the Markov chain $r(t)$ jumps to another state, say $\varsigma$. Therefore, the ecosystem (4) satisfies the SDE

$$
\begin{aligned}
& d x(t) \\
& =\operatorname{diag}\left(x_{1}(t), \ldots, x_{n}(t)\right) \\
& \quad \times[(b(\varsigma)+A(\varsigma) x(t)+B(\varsigma) x(t-\tau)) d t+\sigma(\varsigma) d w(t)],
\end{aligned}
$$

for a random amount of time until the Markov chain $r(t)$ jumps to a new state again.

It should be pointed out that the stochastic population systems under regime switching have received much attention lately. For instance, the stochastic permanence and extinction of a logistic model under regime switching were considered in $[20,24]$, asymptotic results of a competitive Lotka-Volterra model in random environment are obtained in [25], a new single-species model disturbed by both white noise and colored noise in a polluted environment was developed and analyzed in [26], and a general stochastic logistic system under regime switching was proposed and was treated in [27].

In [28], some results have been obtained for (4), such as existence of global positive solutions, stochastically ultimate boundedness, and extinction. In contrast to the existing results, our new contributions in this paper are as follows. (i) The stochastic permanence of solutions is derived.

(ii) The asymptotic estimations of the solutions are obtained, which is related to the stationary probability distribution of the Markov chain.

The rest of the paper is arranged as follows. For convenience of the reader, we briefly recall the main result of [28] in Section 2. The main results of this paper are arranged in Sections 3 and 4 . Section 3 is devoted to the stochastic permanence. The asymptotic estimations of the solutions are obtained in Section 4. Finally, an example is given to illustrate our main results.

\section{Properties of the Solution}

Throughout this paper, unless otherwise specified, let $\left(\Omega, \mathscr{F},\left\{\mathscr{F}_{t}\right\}_{t \geq 0}, P\right)$ be a complete probability space with a filtration $\left\{\mathscr{F}_{t}\right\}_{t \geq 0}$ satisfying the usual conditions (i.e., it is right continuous and $\mathscr{F}_{0}$ contains all $P$-null sets). Let $w(t)$, $t \geq 0$, be a scalar standard Brownian motion defined on this probability space. We also denote by $R_{+}^{n}$ the positive cone in $R^{n}$, that is, $R_{+}^{n}=\left\{x \in R^{n}: x_{i}>0\right.$ for all $\left.1 \leq i \leq n\right\}$ and denote by $\bar{R}_{+}^{n}$ the nonnegative cone in $R^{n}$, that is, $\bar{R}_{+}^{n}=\{x \in$ $R^{n}: x_{i} \geq 0$ for all $\left.1 \leq i \leq n\right\}$. If $A$ is a vector or matrix, its transpose is denoted be $A^{T}$. If $A$ is a matrix, its trace norm is denoted by $|A|=\sqrt{\operatorname{trace}\left(A^{T} A\right)}$, whilst it operator norm is denoted by $\|A\|=\sup \{|A x|:|x|=1\}$. Moreover, let $\tau>0$ and denote by $C\left([-\tau, 0] ; R_{+}\right)$the family of continuous functions from $[-\tau, 0]$ to $R_{+}$.

In this paper we will use a lot of quadratic functions of the form $x^{T} A x$ for the state $x \in R_{+}^{n}$ only. Therefore, for a symmetric $n \times n$ matrix $A$, we naturally introduce the following definition:

$$
\lambda_{\max }^{+}(A)=\sup _{x \in R_{+}^{n},|x|=1} x^{T} A x .
$$

For more properties of $\lambda_{\max }^{+}(A)$, please see the appendix in [7].

Let $r(t)$ be a right-continuous Markov chain on the probability space, taking values in a finite state space $S=$ $\{1,2, \ldots, N\}$, with the generator $\Gamma=\left(\gamma_{u v}\right)$ given by

$$
P\{r(t+\delta)=v \mid r(t)=u\}= \begin{cases}\gamma_{u v} \delta+o(\delta), & \text { if } u \neq v \\ 1+\gamma_{u v} \delta+o(\delta), & \text { if } u=v\end{cases}
$$

where $\delta>0, \gamma_{u v}$ is the transition rate from $u$ to $v$ and $\gamma_{u v} \geq 0$ if $u \neq v$, while $\gamma_{u u}=-\sum_{v \neq u} \gamma_{u v}$. We assume that the Markov chain $r(\cdot)$ is independent of the Brownian motion $w(\cdot)$. It is well known that almost every sample path of $r(\cdot)$ is a rightcontinuous step function with a finite number of jumps in any finite subinterval of $\bar{R}_{+}$. As a standing hypothesis, we assume in this paper that the Markov chain $r(t)$ is irreducible. This is a very reasonable assumption as it means that the system can switch from any regime to any other regime. This is equivalent to the condition that for, any $u, v \in S$, one can find finite numbers $i_{1}, i_{2}, \ldots, i_{k} \in S$ such that $\gamma_{u i_{1}} \gamma_{i_{1} i_{2}}, \ldots, \gamma_{i_{k} v}>0$. 
Under this condition, the Markov chain has a unique stationary (probability) distribution $\pi=\left(\pi_{1}, \pi_{2}, \ldots, \pi_{N}\right) \in R^{1 \times N}$ which can be determined by solving the following linear equation:

$$
\pi \Gamma=0
$$

subject to

$$
\sum_{i=1}^{N} \pi_{i}=1, \quad \pi_{i}>0, \forall i \in S
$$

For the fundamental theory of stochastic differential equations, one can refer to [12, 29].

For convenience and simplicity in the following discussion, for any constant sequence $f_{i}(k),(1 \leq i \leq n, k \in S)$, let

$$
\begin{array}{ll}
\check{f}=\max _{1 \leq i \leq n, k \in S} f_{i}(k), & \check{f}(k)=\max _{1 \leq i \leq n} f_{i}(k), \\
\widehat{f}=\min _{1 \leq i \leq n, k \in S} f_{i}(k), & \widehat{f}(k)=\min _{1 \leq i \leq n} f_{i}(k) .
\end{array}
$$

To proceed, we first state a result, whose proof can be found in [28].

Assumption 1. Assume that there exist positive numbers $c_{1}, \ldots, c_{n}$ such that

$$
\max _{k \in S}\left\{\lambda_{\max }^{+}\left[\frac{1}{2}(\bar{C} A(k)+A(k) \bar{C})\right]\right\}+\max _{k \in S}\|\bar{C} B(k)\| \leq 0,
$$

where $\bar{C}=\operatorname{diag}\left(c_{1}, \ldots, c_{n}\right)$.

Assumption 2. Assume that there exist positive numbers $c_{1}, \ldots, c_{n}$ such that

$$
\max _{k \in S}\left\{\lambda_{\max }^{+}\left[\frac{1}{2}(\bar{C} A(k)+A(k) \bar{C})\right]\right\}+\max _{k \in S}\|\bar{C} B(k)\|<0,
$$

where $\bar{C}=\operatorname{diag}\left(c_{1}, \ldots, c_{n}\right)$.

Assumption 3. Assume that there exist positive numbers $c_{1}, \ldots, c_{n}$ such that

$$
\begin{aligned}
&|c|^{-1} \max _{k \in S}\left\{\lambda_{\max }^{+}\right. {\left.\left[\frac{1}{2}(\bar{C} A(k)+A(k) \bar{C})\right]\right\} } \\
&+\widehat{c}^{-1} \max _{k \in S}\|\bar{C} B(k)\| \leq 0,
\end{aligned}
$$

where $\bar{C}=\operatorname{diag}\left(c_{1}, \ldots, c_{n}\right)$ and $\widehat{c}=\min _{1 \leq i \leq n} c_{i}$.

Theorem 1. (1) Under Assumption 1, for any given initial data $\{x(t):-\tau \leq t \leq 0\} \in C\left([-\tau, 0] ; R_{+}^{n}\right)$, there is a unique solution $x(t)$ to (4) on $t \geq-\tau$ and the solution will remain in $R_{+}^{n}$ with probability 1 , namely, $x(t) \in R_{+}^{n}$ for all $t \geq-\tau$ almost surely.

(2) Under Assumption 2, for any given initial data $\{x(t)$ : $-\tau \leq t \leq 0\} \in C\left([-\tau, 0] ; R_{+}^{n}\right)$ and any given positive constant $p$, there are two positive constant $K_{1}(p)$ and $K_{2}(p)$, such that the solution $x(t)$ of $(4)$ has the properties that

$$
\begin{gathered}
\limsup _{t \rightarrow \infty} E|x(t)|^{p} \leq K_{1}(p), \\
\limsup _{t \rightarrow \infty} \frac{1}{t} \int_{0}^{t} E|x(s)|^{p+1} d s \leq K_{2}(p) .
\end{gathered}
$$

(3) Solutions of (4) are stochastically ultimately bounded under Assumption 2; that is, for any $\varepsilon \in(0,1)$, there exists a positive constants $H=H(\varepsilon)$, such that the solutions of (4) with any positive initial value have the property that

$$
\limsup _{t \rightarrow+\infty} P\{|x(t)|>H\}<\varepsilon .
$$

(4) Under Assumption 3, for any given initial data $\{x(t)$ : $-\tau \leq t \leq 0\} \in C\left([-\tau, 0] ; R_{+}\right)$, the solution $x(t)$ of $(4)$ has the properties that

$$
\limsup _{t \rightarrow \infty} \frac{1}{t} \log |x(t)| \leq \sum_{i=1}^{n} \pi_{k} \beta(k) \quad \text { a.s., }
$$

where $\beta(k)=\check{b}(k)-(1 / 2) \widehat{\sigma}^{2}(k)$. Particularly, if $\sum_{k=1}^{N} \pi_{k} \beta(k)<$ 0 , then

$$
\limsup _{t \rightarrow \infty} \frac{1}{t} \log |x(t)|<0 \quad \text { a.s. }
$$

That is, the population will become extinct exponentially with probability 1.

\section{Stochastic Permanence}

Definition 2. Equation (4) is said to be stochastically permanent if, for any $\varepsilon \in(0,1)$, there exist positive constants $H=H(\varepsilon), \delta=\delta(\varepsilon)$ such that

$$
\begin{aligned}
& \liminf _{t \rightarrow+\infty} P\{|x(t)| \leq H\} \geq 1-\varepsilon, \\
& \liminf _{t \rightarrow+\infty} P\{|x(t)| \geq \delta\} \geq 1-\varepsilon,
\end{aligned}
$$

where $x(t)$ is the solution of (4) with any positive initial value.

It is obvious that if a stochastic equation is stochastically permanent, its solutions must be stochastically ultimately bounded. For convenience, let

$$
\alpha(k)=\widehat{b}(k)-\frac{1}{2} \check{\sigma}^{2}(k), \quad \beta(k)=\check{b}(k)-\frac{1}{2} \widehat{\sigma}^{2}(k),
$$

and we impose the following assumptions.

Assumption 4. For some $u \in S, \gamma_{i u}>0$ (for all $\left.i \neq u\right)$.

Assumption 5. $\sum_{k=1}^{N} \pi_{k} \alpha(k)>0$.

Assumption 6. For each $k \in S, \alpha(k)>0$.

Let $G$ be a vector or matrix. By $G \gg 0$, we mean all elements of $G$ are positive, and by $G \geq 0$, we mean all elements of $G$ are nonnegative. We also adopt here the traditional notation by letting

$$
Z^{N \times N}=\left\{A=\left(a_{i j}\right)_{N \times N}: a_{i j} \leq 0, i \neq j\right\} .
$$


Lemma 3 (see [29]). If $A=\left(a_{i j}\right)_{N \times N} \in Z^{N \times N}$ has all of its row sums positive, that is,

$$
\sum_{j=1}^{N} a_{i j}>0, \quad \forall 1 \leq i \leq N
$$

then $A>0$.

Lemma 4 (see [29]). If $A \in Z^{N \times N}$, then the following statements are equivalent:

(1) $A$ is a nonsingular $M$-matrix.

(2) All of the principal minors of $A$ are positive; that is,

$$
\left|\begin{array}{ccc}
a_{11} & \cdots & a_{1 k} \\
\vdots & \vdots & \vdots \\
a_{1 k} & \cdots & a_{k k}
\end{array}\right|>0 \quad \text { for every } k=1,2, \ldots, N
$$

(3) A is semipositive; that is, there exists $x \gg 0$ in $R^{N}$ such that $A x \gg 0$.

Lemma 5 (see [23]). Assumptions 4 and 5 imply that there exists a constant $\theta>0$ such that the matrix

$$
A(\theta)=\operatorname{diag}\left(\xi_{1}(\theta), \xi_{2}(\theta), \ldots, \xi_{N}(\theta)\right)-\Gamma
$$

is a nonsingular M-matrix, where

$$
\xi_{k}(\theta)=\theta \alpha(k)-\frac{1}{2} \theta^{2} \check{\sigma}^{2}(k), \quad \forall k \in S
$$

Lemma 6 (see [23]). Assumption 6 implies that there exists a constant $\theta>0$ such that the matrix $A(\theta)$ is a nonsingular $M$ matrix.

Lemma 7. If there exists a constant $\theta>0$ such that $A(\theta)$ is a nonsingular $M$-matrix and $B(k) \geq 0(k=1,2, \ldots, N)$, then the global positive solution $x(t)$ of $(4)$ has the property that

$$
\limsup _{t \rightarrow \infty} E\left(\frac{1}{|x(t)|^{\theta}}\right) \leq H
$$

where $H$ is a fixed positive constant (defined by (42) in the proof).

Proof. Define $V(x)=\sum_{i=1}^{n} x_{i}$ on $x \in R_{+}^{n}$. Then

$$
\begin{aligned}
d V(x(t))=x^{T}(t)[ & (b(r(t))+A(r(t)) x(t) \\
& +B(r(t)) x(t-\tau)) d t \\
& +\sigma(r(t)) d w(t)] .
\end{aligned}
$$

Define also

$$
U(x)=\frac{1}{V(x)} \quad \text { on } x \in R_{+}^{n}
$$

Let $y(t)=x(t-\tau)$. Applying the generalized Itô formula, we derive from (28) that

$$
\begin{aligned}
d U= & -U^{2} d V+U^{3}(d V)^{2} \\
= & -U^{2} x^{T}\{[b(r(t))+A(r(t)) x+B(r(t)) y] d t \\
& \quad+\sigma(r(t)) d w(t)\}+U^{3}\left|x^{T} \sigma(r(t))\right|^{2} d t \\
= & \left\{-U^{2} x^{T}[b(r(t))+A(r(t)) x+B(r(t)) y]\right. \\
& \left.\quad+U^{3}\left|x^{T} \sigma(r(t))\right|^{2}\right\} d t-U^{2} x^{T} \sigma(r(t)) d w(t)
\end{aligned}
$$

dropping $x(t)$ from $U(x(t)), V(x(t))$ and $t$ from $x(t), y(t)$, respectively. By Lemma 4 , for given $\theta$, there is a vector $\vec{q}=$ $\left(q_{1}, q_{2}, \ldots, q_{N}\right)^{T} \gg 0$ such that

$$
\vec{\lambda}=\left(\lambda_{1}, \lambda_{2}, \ldots, \lambda_{N}\right)^{T}:=A(\theta) \vec{q} \gg 0
$$

namely,

$$
q_{k}\left(\theta \alpha(k)-\frac{1}{2} \theta^{2} \check{\sigma}^{2}(k)\right)-\sum_{l=1}^{N} \gamma_{k l} q_{l}>0 \quad \forall 1 \leq k \leq N
$$

Define the function $\bar{V}: R_{+} \times S \rightarrow R_{+}$by $\bar{V}(U, k)=$ $q_{k}(1+U)^{\theta}$. It follows from the generalized Itô formula that

$$
\begin{aligned}
E \bar{V}(U(t), r(t))= & \bar{V}(U(0), r(0)) \\
& +E \int_{0}^{t} L \bar{V}(U(s), x(s-\tau), r(s)) d s,
\end{aligned}
$$

where

$$
\begin{aligned}
& L \bar{V}(U, x, y, k) \\
& =q_{k} \theta(1+U)^{\theta-1}\left\{-U^{2} x^{T}[b(k)+A(k) x+B(k) y]\right. \\
& \left.+U^{3}\left|x^{T} \sigma(k)\right|^{2}\right\}+q_{k} \frac{\theta(\theta-1)}{2} \\
& \times(1+U)^{\theta-2} U^{4}\left|x^{T} \sigma(k)\right|^{2}+\sum_{l=1}^{N} \gamma_{k l} q_{l}(1+U)^{\theta} \\
& =q_{k} \theta(1+U)^{\theta-2} \\
& \times\left\{-(1+U) U^{2} x^{T}[b(k)+A(k) x+B(k) y]\right. \\
& \left.+(1+U) U^{3}\left|x^{T} \sigma(k)\right|^{2}+\frac{1}{2}(\theta-1) U^{4}\left|x^{T} \sigma(k)\right|^{2}\right\} \\
& +\sum_{l=1}^{N} \gamma_{k l} q_{l}(1+U)^{\theta}
\end{aligned}
$$


It is easy to see that, for all $x \in R_{+}^{n}$,

$$
\begin{gathered}
-\frac{x^{T} A(k) x}{V^{2}} \leq K, \\
-\frac{x^{T} b(k)}{V}+\frac{\left|x^{T} \sigma(k)\right|^{2}-x^{T} A(k) x}{V^{2}} \leq K,
\end{gathered}
$$

where $K$ is a positive constant, while

$$
\begin{aligned}
\frac{x^{T} b(k)}{V}-\frac{1}{2}(\theta+1) \frac{\left|x^{T} \sigma(k)\right|^{2}}{V^{2}} & \geq \widehat{b}(k)-\frac{1}{2}(\theta+1) \check{\sigma}^{2}(k) \\
& =\widehat{\beta}(k)-\frac{1}{2} \theta \check{\sigma}^{2}(k) .
\end{aligned}
$$

Consequently,

$$
\begin{aligned}
-(1+U) & U^{2} x^{T}[b(k)+A(k) x+B(k) y] \\
& +(1+U) U^{3}\left|x^{T} \sigma(k)\right|^{2}+\frac{1}{2}(\theta-1) U^{4}\left|x^{T} \sigma(k)\right|^{2} \\
= & -U^{2} x^{T} b(k)-U^{3} x^{T} b(k)-U^{2} x^{T} A(k) x \\
& -U^{3} x^{T} A(k) x-U^{2} x^{T} B(k) y-U^{3} x^{T} B(k) y \\
& +U^{3}\left|x^{T} \sigma(k)\right|^{2}+\frac{1}{2}(\theta+1) U^{4}\left|x^{T} \sigma(k)\right|^{2} \\
\leq & -\left(\frac{x^{T} b(k)}{V}-\frac{1}{2}(\theta+1) \frac{\left|x^{T} \sigma(k)\right|^{2}}{V^{2}}\right) U^{2} \\
& +\left(-\frac{x^{T} b(k)}{V}+\frac{\left|x^{T} \sigma(k)\right|^{2}-x^{T} A(k) x}{V^{2}}\right) U \\
& -\frac{x^{T} A(k) x}{V^{2}} \\
\leq & \left(\alpha(k)-\frac{1}{2} \theta \check{\sigma}^{2}(k)\right) U^{2}+K(1+U) .
\end{aligned}
$$

Substituting (37) into (34) yields

$$
\begin{aligned}
& L \bar{V}(U, x, y, k) \\
& \leq q_{k} \theta(1+U)^{\theta-2}\left\{-\left(\alpha(k)-\frac{1}{2} \theta \check{\sigma}^{2}(k)\right) U^{2}+K(1+U)\right\} \\
& \quad+\sum_{l=1}^{N} \gamma_{k l} q_{l}(1+U)^{\theta} \\
& =(1+U)^{\theta-2}
\end{aligned}
$$

$$
\begin{gathered}
\times\left\{-\left[q_{k} \theta\left(\alpha(k)-\frac{1}{2} \theta \check{\sigma}^{2}(k)\right)-\sum_{l=1}^{N} \gamma_{k l} q_{l}\right] U^{2}\right. \\
+\left(q_{k} \theta K+2 \sum_{l=1}^{N} \gamma_{k l} q_{l}\right) U \\
\left.+\left(q_{k} \theta K+2 \sum_{l=1}^{N} \gamma_{k l} q_{l}\right)\right\} .
\end{gathered}
$$

Now, choose a constant $\kappa>0$ sufficiently small such that it satisfies $\vec{\lambda}-\kappa \vec{q} \gg 0$, that is,

$$
q_{k}\left(\theta \alpha(k)-\frac{1}{2} \theta^{2} \check{\sigma}^{2}(k)\right)-\sum_{l=1}^{N} \gamma_{k l} q_{l}-\kappa q_{k}>0 \quad \forall 1 \leq k \leq N .
$$

Then, by the generalized Itô formula again,

$$
\begin{aligned}
& E\left[e^{\kappa t} \bar{V}(U(t), r(t))\right] \\
& =\bar{V}(U(0), r(0)) \\
& \quad+E \int_{0}^{t}\left[\kappa e^{\kappa t} \bar{V}(U(s), r(s))\right. \\
& \left.\quad+e^{\kappa t} L \bar{V}(U(s), x(s), x(s-\tau), r(s))\right] d s .
\end{aligned}
$$

It is computed that

$$
\begin{aligned}
& \kappa e^{\kappa t} \bar{V}(U, i)+e^{\kappa t} L \bar{V}(U, x, y, i) \\
& \leq e^{\kappa t}(1+U)^{\theta-2} \\
& \times\left\{\kappa q_{k}(1+U)^{2}\right. \\
& -\left[q_{k} \theta\left(\alpha(k)-\frac{1}{2} \theta \check{\sigma}^{2}(k)\right)-\sum_{l=1}^{N} \gamma_{k l} q_{l}\right] U^{2} \\
& \left.+\left(q_{k} \theta K+2 \sum_{l=1}^{N} \gamma_{k l} q_{l}\right) U+\left(q_{k} \theta K+2 \sum_{l=1}^{N} \gamma_{k l} q_{l}\right)\right\} \\
& \leq e^{\kappa t}(1+U)^{\theta-2} \\
& \times\left\{-\left[q_{k} \theta\left(\alpha(k)-\frac{1}{2} \theta \check{\sigma}^{2}(k)\right)-\sum_{l=1}^{N} \gamma_{k l} q_{l}-\kappa q_{k}\right] U^{2}\right. \\
& +\left(q_{k} \theta K+2 \sum_{l=1}^{N} \gamma_{k l} q_{l}+2 \kappa q_{k}\right) U \\
& \left.+\left(q_{k} \theta K+2 \sum_{l=1}^{N} \gamma_{k l} q_{l}+\kappa q_{k}\right)\right\} \\
& \leq n^{-\theta} \widehat{q} \kappa H e^{\kappa t},
\end{aligned}
$$


where

$$
\begin{aligned}
& H=\frac{1}{\widehat{q} \kappa} n^{\theta} \max _{i \in S}\left\{\sup _{U \in R_{+}}(1+U)^{\theta-2}\right. \\
& \times\left\{-\left[q_{k} \theta\left(\alpha(k)-\frac{1}{2} \theta \check{\sigma}^{2}(k)\right)\right.\right. \\
& \left.-\sum_{l=1}^{N} \gamma_{k l} q_{l}-\kappa q_{k}\right] U^{2} \\
& +\left(q_{k} \theta K+2 \sum_{l=1}^{N} \gamma_{k l} q_{l}+2 \kappa q_{k}\right) U \\
& \left.\left.+\left(q_{k} \theta K+2 \sum_{l=1}^{N} \gamma_{k l} q_{l}+\kappa q_{k}\right)\right\} \vee 1\right\}
\end{aligned}
$$

This implies

$$
\limsup _{t \rightarrow \infty} E\left[U^{\theta}(x(t))\right] \leq \limsup _{t \rightarrow \infty} E\left[(1+U(x(t)))^{\theta}\right] \leq n^{-\theta} H .
$$

For $x(t) \in R_{+}^{n}$, note that $\left(\sum_{i=1}^{n} x_{i}(t)\right)^{\theta} \leq\left(n \max _{1 \leq i \leq n} x_{i}(t)\right)^{\theta} \leq$ $n^{\theta}|x(t)|^{\theta}$. Consequently,

$$
\limsup _{t \rightarrow \infty} E\left(\frac{1}{|x(t)|^{\theta}}\right) \leq H .
$$

The required assertion (27) is obtained.

Assumption 7. Assume that there exist positive numbers $c_{1}, \ldots, c_{n}$ such that

$$
\max _{k \in S}\left\{\lambda_{\max }^{+}\left[\frac{1}{2}(\bar{C} A(k)+A(k) \bar{C})\right]\right\}+\max _{k \in S}\|\bar{C} B(k)\|<0,
$$

where $\bar{C}=\operatorname{diag}\left(c_{1}, \ldots, c_{n}\right)$. Moreover for each $k \in S, B(k) \geq 0$.

Theorem 8. Under Assumptions 4, 5, and 7, (4) is stochastically permanent.

The proof is a simple application of the Chebyshev's inequality, Lemmas 5 and 7, and Theorem 1(3). Similarly, we have the following result.

Theorem 9. Under Assumptions 6 and 7, (4) is stochastically permanent.

\section{Asymptotic Properties}

Lemma 10. Under Assumption 2, for any given initial data $\{x(t):-\tau \leq t \leq 0\} \in C\left([-\tau, 0] ; R_{+}\right)$, the solution $x(t)$ of (4) with any positive initial value has the property

$$
\limsup _{t \rightarrow \infty} \frac{\log (x(t))}{\log t} \leq 1 \quad \text { a.s. }
$$

Proof. By Theorem 1 (1), the solution $x(t)$ will remain in $R_{+}^{n}$ for all $t \geq-\tau$ with probability 1 . Denote $V(x)=\sum_{i=1}^{n} x_{i}$, on $x \in R_{+}^{n}$. It is known that

$$
\begin{aligned}
& d V(x(t)) \\
& =x^{T}(t)[(b(r(t))+A(r(t)) x(t)+B(r(t)) x(t-\tau)) d t \\
& \quad+\sigma(r(t)) d w(t)] .
\end{aligned}
$$

We can also derive from this that

$$
\begin{aligned}
& E\left(\sup _{t \leq u \leq t+1} V(x(u))\right) \\
& \leq E V(x(t))+\max _{k \in S}|b(k)| \int_{t}^{t+1} E|x(s)| d s \\
&+\max _{k \in S}|A(k)| \int_{t}^{t+1} E|x(s)|^{2} d s+\max _{k \in S}|B(k)| \\
& \times \int_{t}^{t+1} E(|x(s)||x(s-\tau)|) d s \\
&+E\left(\sup _{t \leq u \leq t+1} \int_{t}^{u} x^{T}(s) \sigma(r(s)) d w(s)\right) .
\end{aligned}
$$

From (15), we know that $\limsup _{t \rightarrow \infty} E|x(t)| \leq K_{1}(1)$ and $\lim \sup _{t \rightarrow \infty} E|x(t)|^{2} \leq K_{1}(2)$. By the well-known BDG's inequality [29] and the Hölder's inequality, we derive that

$$
\begin{aligned}
E\left(\sup _{t \leq u \leq t+1} \int_{t}^{u} x^{T}(s) \sigma(r(s)) d w(s)\right) \\
\quad \leq 3 \max _{k \in S}|b(k)| E\left(\int_{t}^{t+1}|x(s)|^{2} d s\right)^{1 / 2} \\
\leq E\left(9 \check{\sigma} \int_{t}^{t+1} x^{2}(s) d s\right)^{1 / 2} \\
\leq E\left(\sup _{t \leq u \leq t+1} x(u) \cdot 9 \check{\sigma} \int_{t}^{t+1} x(s) d s\right)^{1 / 2} \\
\quad \leq E\left[\left(\frac{1}{2} \sup _{t \leq u \leq t+1} x(u)\right)^{2}+9 \check{\sigma} \int_{t}^{t+1}(x(s) d s)^{2}\right]^{1 / 2} \\
\leq E\left(\frac{1}{2} \sup _{t \leq u \leq t+1} x(u)+9 \check{\sigma}^{2} \int_{t}^{t+1} x(s) d s\right) \\
\leq \frac{1}{2} E\left(\sup _{t \leq u \leq t+1} x(u)\right)+9 \check{\sigma}^{2} \int_{t}^{t+1} E(x(s)) d s .
\end{aligned}
$$

Combining the inequality above with

$$
\begin{aligned}
& \int_{t}^{t+1} E(|x(s)||x(s-\tau)|) d s \\
& \quad \leq \frac{1}{2} \int_{t}^{t+1} E|x(s)|^{2} d s+\frac{1}{2} \int_{t}^{t+1} E|x(s-\tau)|^{2} d s,
\end{aligned}
$$


we get that

$$
\begin{aligned}
& E\left(\sup _{t \leq u \leq t+1} V(x(u))\right) \\
& \leq E V x(t)+\max _{k \in S}|b(k)| \int_{t}^{t+1} E|x(s)| d s \\
&+\left(\max _{k \in S}|A(k)|+\frac{1}{2} \max _{k \in S}|B(k)|\right) \int_{t}^{t+1} E|x(s)|^{2} d s \\
&+\frac{1}{2} \max _{k \in S}|B(k)| \int_{t}^{t+1} E|x(s-\tau)|^{2} d s \\
&+3 \max _{k \in S}|b(k)| E\left(\int_{t}^{t+1}|x(s)|^{2} d s\right)^{1 / 2} .
\end{aligned}
$$

Recalling the following inequality $|x| \leq \sum_{i=1}^{n} x_{i} \leq V(x)$ for any $x \in R_{+}^{n}$, we obtain

$$
\begin{aligned}
& \limsup _{t \rightarrow \infty} E\left(\sup _{t \leq u \leq t+1} x(u)\right) \\
& \leq \max _{k \in S}|b(k)| K_{1}(1) \\
& \quad+\left(\max _{k \in S}|A(k)|+\max _{k \in S}|B(k)|\right) K_{1}(2) \\
& \quad+3 \max _{k \in S}|b(k)|(K(2))^{1 / 2} .
\end{aligned}
$$

It is following from (52) that there is a positive constant $M$ such that

$$
E\left(\sup _{k \leq t \leq k+1}|x(t)|\right) \leq M, \quad k=1,2, \ldots
$$

Let $\varepsilon>0$ be arbitrary. Then, by Chebyshev's inequality, we have

$$
P\left(\sup _{k \leq t \leq k+1}|x(u)|>k^{1+\varepsilon}\right) \leq \frac{M}{k^{1+\varepsilon}}, \quad k=1,2, \ldots
$$

Applying the well-known Borel-Cantelli lemma [24], we obtain that for almost all $\omega \in \Omega$

$$
\sup _{k \leq t \leq k+1}|x(u)| \leq k^{1+\varepsilon}
$$

holds for all but finitely many $k$. Hence, there exists a $k_{0}(\omega)$, for almost all $\omega \in \Omega$, for which (55) holds whenever $k \geq k_{0}$. Consequently, for almost all $\omega \in \Omega$, if $k \geq k_{0}$ and $k \leq t \leq k+1$,

$$
\frac{\log (|x(t)|)}{\log t} \leq \frac{(1+\varepsilon) \log k}{\log k}=1+\varepsilon .
$$

Therefore, $\lim \sup _{t \rightarrow \infty}(\log (|x(t)|) / \log t) \leq 1+\varepsilon$ a.s. Letting $\varepsilon \rightarrow 0$, we obtain the desired assertion (46).

Lemma 11. If there exists a constant $\theta>0$ such that $A(\theta)$ is a nonsingular $M$-matrix and for each $k \in S, B(k) \geq 0$, then the global positive solution $x(t)$ of SDE (4) has the property that

$$
\liminf _{t \rightarrow \infty} \frac{\log (|x(t)|)}{\log t} \geq-\frac{1}{\theta} \quad \text { a.s. }
$$

Proof. Let $U: R_{+}^{n} \rightarrow R_{+}^{n}$ be the same as defined by (29); for convenience, we write $U(x(t))=U(t)$. Applying the generalized Itô formula, for the fixed constant $\theta>0$, we derive from (37) that

$$
\begin{aligned}
d(1+U(t))^{\theta} \leq & \theta(1+U(t))^{\theta-2} \\
\times & \times-U^{2}(t)\left(\alpha(r(t))-\frac{1}{2} \theta \check{\sigma}^{2}(r(t))\right) \\
& \left.\quad+K_{1} U(t)+K_{1}+\check{b}\right] d t \\
& -\theta(1+U(t))^{\theta-1} U^{2}(t) \sigma(r(t)) d w(t) .
\end{aligned}
$$

By (43), there exists a positive constant $M$ such that

$$
E(1+U(t))^{\theta} \leq M \quad \text { on } t \geq 0
$$

Let $\delta>0$ be sufficiently small such that

$$
\theta\left[\left(|\widehat{\alpha}|+\frac{1}{2} \theta \check{\sigma}^{2}+K_{1}\right) \delta+3 \max _{k \in S}|\sigma(k)| \delta^{1 / 2}\right]<\frac{1}{2}
$$

Then (58) implies that

$$
\begin{aligned}
& E\left[\sup _{(k-1) \delta \leq t \leq k \delta}(1+U(t))^{\theta}\right] \\
& \leq E\left[(1+U((k-1) \delta))^{\theta}\right] \\
& +E\left\{\sup _{(k-1) \delta \leq t \leq k \delta} \mid \int_{(k-1) \delta}^{t} \theta(1+U(s))^{\theta-2}\right. \\
& \times\left[-U^{2}(s)(\widehat{\alpha}(r(s))\right. \\
& +E\left\{\sup _{(k-1) \delta \leq t \leq k \delta} \mid \int_{(k-1) \delta}^{t} \theta(1+U(s))^{\theta-1} U^{2}\right. \\
& \left.\left.+K_{1}(U(s)+1)\right] d s \mid\right\} \\
& \left.\times(s) x^{T}(s) \sigma(r(s)) d w(s) \mid\right\}
\end{aligned}
$$


It is computed that

$$
\begin{aligned}
& E\left\{\sup _{(k-1) \delta \leq t \leq k \delta} \mid \int_{(k-1) \delta}^{t} \theta(1+U(s))^{\theta-2}\right. \\
& \quad \times\left[-U^{2}(s)\left(\widehat{\alpha}(r(s))-\frac{1}{2} \theta \check{\sigma}^{2}(r(s))\right)\right. \\
& \left.\left.+K_{1}(U(s)+1)\right] d s \mid\right\} \\
& \leq E\left\{\int_{(k-1) \delta}^{k \delta} \mid \theta(1+U(s))^{\theta-2}\left[-U^{2}(s)\left(\widehat{\alpha}-\frac{1}{2} \theta \check{\sigma}^{2}\right)\right.\right. \\
& \leq \theta E\left\{\int_{(k-1) \delta}^{k \delta}\left(|\widehat{\alpha}|+\frac{1}{2} \theta \check{\sigma}^{2}+K_{1}\right)(1+U(s))^{\theta} d s\right\} \\
& \left.\leq \theta\left(|\widehat{\alpha}|+\frac{1}{2} \theta \check{\sigma}^{2}+K_{1}\right) E\left[\int_{(k-1) \delta(k-1) \delta \leq s \leq k \delta}^{t} \quad+K_{1}(U(s)+1)\right] \mid d s\right\} \\
& \leq \theta\left(|\widehat{\alpha}|+\frac{1}{2} \theta \check{\sigma}^{2}+K_{1}\right) \delta E\left[\begin{array}{l}
\left.\sup _{(k-1) \delta \leq t \leq k \delta}(1+U(t))^{\theta}\right] .
\end{array}\right]
\end{aligned}
$$

On the other hand, by the BDG's inequality, we derive that

$$
\begin{aligned}
& E\left\{\sup _{(k-1) \delta \leq t \leq k \delta} \mid \int_{(k-1) \delta}^{k \delta} \theta(1+U(s))^{\theta-1} U^{2}\right. \\
& \left.\times(s) x^{T}(s) \sigma(r(s)) d w(s) \mid\right\} \\
& \leq 3 E\left\{\int_{(k-1) \delta}^{k \delta}\left[\theta(1+U(s))^{\theta-1} U^{2}(s)\right]^{2}\left|x^{T} \sigma(r(s))\right|^{2}\right\}^{1 / 2} \\
& \leq 3 \theta E\left\{\int_{(k-1) \delta}^{k \delta}(1+U(s))^{2(\theta-1)} U^{2}(s) \frac{|x(s)|^{2}|\sigma(r(s))|^{2}}{|x(s)|^{2}}\right\}^{1 / 2} \\
& \leq 3 \theta \max _{k \in S}|\sigma(k)| E\left\{\int_{(k-1) \delta}^{k \delta}(1+U(s))^{2 \theta} d s\right\}^{1 / 2} \\
& \leq 3 \theta \max _{k \in S}|\sigma(k)| \delta^{1 / 2} E\left\{\sup _{(k-1) \delta \leq t \leq k \delta}(1+U(s))^{2 \theta}\right\}^{1 / 2} \\
& \leq 3 \theta \max _{k \in S}|\sigma(k)| \delta^{1 / 2} E\left\{\sup _{(k-1) \delta \leq t \leq k \delta}(1+U(s))^{\theta}\right\} \text {. }
\end{aligned}
$$

Substituting this and (62) into (61) gives

$$
\begin{aligned}
& E\left[\sup _{(k-1) \delta \leq t \leq k \delta}(1+U(t))^{\theta}\right] \\
& \leq E\left[(1+U((k-1) \delta))^{\theta}\right] \\
& +\theta\left\{\left[\widehat{\alpha}+\frac{1}{2} \theta \check{\sigma}^{2}+K_{1}\right] \delta+3 \max _{k \in S}|\sigma(k)| \delta^{1 / 2}\right\} \\
& \quad \times E\left\{\sup _{(k-1) \delta \leq t \leq k \delta}(1+U(s))^{\theta}\right\} .
\end{aligned}
$$

Making use of (59) and (60), we obtain that

$$
\sup _{(k-1) \delta \leq t \leq k \delta} E\left[(1+U(t))^{\theta}\right] \leq 2 M \quad \text { on } t \geq 0 .
$$

Let $\varepsilon>0$ be arbitrary. Then, by Chebyshev inequality, we have

$$
\begin{aligned}
& P\left\{\omega: \sup _{(k-1) \delta \leq t \leq k \delta}(1+U(t))^{\theta}>(k \delta)^{1+\varepsilon}\right\} \\
& \leq \frac{2 M}{(k \delta)^{1+\varepsilon}}, \quad k=1,2, \ldots
\end{aligned}
$$

Applying the Borel-Cantelli lemma, we obtain that for almost all $\omega \in \Omega$

$$
\sup _{(k-1) \delta \leq t \leq k \delta}(1+U(t))^{\theta} \leq(k \delta)^{1+\varepsilon}
$$

holds for all but finitely many $k$. Hence, there exists an integer $k_{0}(\omega)>1 / \delta+2$, for almost all $\omega \in \Omega$, for which (67) holds whenever $k \geq k_{0}$. Consequently, for almost all $\omega \in \Omega$, if $k \geq$ $k_{0}$ and $(k-1) \delta \leq t \leq k \delta$,

$$
\frac{\log (1+U(t))^{\theta}}{\log t} \leq \frac{(1+\varepsilon) \log (k \delta)}{\log ((k-1) \delta)} \leq 1+\varepsilon .
$$

Therefore $\lim \sup _{t \rightarrow \infty}\left(\log (1+U(t))^{\theta} / \log t\right) \leq 1+\varepsilon$ a.s. Let $\varepsilon \rightarrow 0$, we obtain the desired assertion

$$
\limsup _{t \rightarrow \infty} \frac{\log (1+U(t))^{\theta}}{\log t} \leq 1 \text { a.s. }
$$

Recalling the definition of $U(t)$, this yields $\limsup _{t \rightarrow \infty}\left(\log |x(t)|^{-\theta} / \log t\right) \leq 1$ a.s., which further implies

$$
\liminf _{t \rightarrow \infty} \frac{\log (|x(t)|)}{\log t} \geq-\frac{1}{\theta} \quad \text { a.s. }
$$

This is our required assertion (57).

Assumption 8. Assume that there exist positive numbers $c_{1}, \ldots, c_{n}$ such that

$$
\begin{aligned}
-\lambda= & |c|^{-1} \max _{k \in S}\left\{\lambda_{\max }^{+}\left[\frac{1}{2}(\bar{C} A(k)+A(k) \bar{C})\right]\right\} \\
& +\widehat{c}^{-1} \max _{k \in S}\|\bar{C} B(k)\|<0,
\end{aligned}
$$

where $\bar{C}=\operatorname{diag}\left(c_{1}, \ldots, c_{n}\right)$ and $\widehat{c}=\min _{1 \leq i \leq n} c_{i}$. Moreover for each $k \in S, B(k) \geq 0$. 
Theorem 12. Under Assumptions 4, 5, and 8, for any given initial data $\{x(t):-\tau \leq t \leq 0\} \in C\left([-\tau, 0] ; R_{+}\right)$, the solution $x(t)$ of (4) obeys

$$
\begin{aligned}
& \limsup _{t \rightarrow \infty} \frac{1}{t} \int_{0}^{t}|x(s)| d s \leq \frac{1}{\lambda} \sum_{i=k}^{N} \pi_{k} \beta(k) \quad \text { a.s., } \\
& \liminf _{t \rightarrow \infty} \frac{1}{t} \int_{0}^{t}|x(s)| d s \geq \frac{2 \widehat{c}}{\widehat{\lambda}} \sum_{k=1}^{n} \pi_{k} \alpha(k) \quad \text { a.s., }
\end{aligned}
$$

where $-\widehat{\lambda}=\min _{k \in S}\left[\lambda_{\min }\left(\bar{C} A(k)+A^{T}(k) \bar{C}\right)\right]<0$.

Proof. By Theorem 1(1), the solution $x(t)$ will remain in $R_{+}$for all $t \geq-\tau$ with probability 1 . Define $V(x)=c^{T} x=\sum_{i=1}^{n} c_{i} x_{i}$, for $x \in R_{+}^{n}$. By generalized Itô formula, one has

$$
\begin{aligned}
d V(x(t))=x^{T}(t) \bar{C}[ & (b(r(t))+A(r(t)) x(t) \\
& +B(r(t)) x(t-\tau)) d t \\
& +\sigma(r(t)) d w(t)] .
\end{aligned}
$$

From Lemmas 5, 10, and 11, it follows that

$$
\lim _{t \rightarrow \infty} \frac{\log (V(x(t)))}{t}=0 \quad \text { a.s. }
$$

By (73), it has

$$
\begin{gathered}
d \log V(x(t)) \\
=V^{-1}(x(t)) x^{T}(t) \bar{C}[(b(r(t))+A(r(t)) x(t) \\
+B(r(t)) x(t-\tau)) d t \\
+\sigma(r(t)) d w(t)] \\
-0.5 V^{-2}(x(t))\left|x^{T}(t) \bar{C} \sigma(r(t))\right|^{2} d t .
\end{gathered}
$$

Meanwhile,

$$
\begin{aligned}
& \alpha(r(t)) \leq V^{-1}(x(t)) \bar{C} b(r(t)) \\
& -0.5 V^{-2}(x(t))\left|x^{T}(t) \bar{C} \sigma(r(t))\right|^{2} \\
& \leq \beta(r(t)), \\
& -0.5 \widehat{c}^{-1} \widehat{\lambda}|x(t)| \\
& \leq V^{-1}(x(t)) x^{T}(t) \bar{C}(A(r(t)) x(t) \\
& +B(r(t)) x(t-\tau)) \\
& \leq|c|^{-1} \max _{k \in S}\left\{\lambda_{\max }^{+}\left[\frac{1}{2}\left(\bar{C} A(k)+A^{T}(k) \bar{C}\right)\right]\right\} \\
& +\widehat{c}^{-1} \max _{k \in S}\|\bar{C} B(k)\||x(t)|+0.5 \widehat{c}^{-1} \max _{k \in S}\|\bar{C} B(k)\| \\
& \times(-|x(t)|+|x(t-\tau)|) .
\end{aligned}
$$

Substituting (76) into (75) yields

$$
\begin{aligned}
d \log V(x(t)) \leq & \beta(r(t)) d t-\lambda|x(t)| d t \\
& +0.5 \widehat{c}^{-1} \max _{k \in S}\|\bar{C} B(k)\|(-|x(t)|+|x(t-\tau)|) \\
& +V^{-1}(x(t)) x^{T}(t) \bar{C} \sigma(r(t)) d w(t) .
\end{aligned}
$$

Hence,

$$
\begin{aligned}
\log V(x(t))+\lambda \int_{0}^{t}|x(s)| d s \\
\leq \log V(x(0))+\int_{0}^{t} \beta(r(s)) d s+\int_{-\tau}^{0}|x(s)| d s \\
\quad+\int_{0}^{t} V^{-1}(x(s)) x^{T}(s) \bar{C} \sigma(r(s)) d w(s) .
\end{aligned}
$$

Applying the strong law of large numbers for martingales, we have

$$
\lim _{t \rightarrow \infty} \frac{1}{t} \int_{0}^{t} V^{-1}(x(s)) x^{T}(s) \bar{C} \sigma(r(s)) d w(s)=0 \quad \text { a.s. }
$$

Dividing both sides of (78) by $t$ and letting $t \rightarrow \infty$, we obtain that

$$
\lambda \limsup _{t \rightarrow+\infty} \frac{1}{t} \int_{0}^{t}|x(s)| d s \leq \sum_{k=1}^{N} \pi_{k} \beta(k) \quad \text { a.s., }
$$

which implies the required assertion (72).

On the other hand, it is observed from (75)-(76) that

$$
\begin{aligned}
d \log V(x(t)) \geq & \alpha(r(t)) d t-0.5 \widehat{c}^{-1} \hat{\lambda}|x(t)| d t \\
& +V^{-1}(x(t)) x^{T}(t) \bar{C} \sigma(r(t)) d w(t) .
\end{aligned}
$$

Hence,

$$
\begin{aligned}
\log V(x(t))+0.5 \widehat{c}^{-1} \widehat{\lambda} \int_{0}^{t}|x(s)| d s \\
\geq \log V(x(0))+\int_{0}^{t} \alpha(r(s)) d s \\
\quad+\int_{0}^{t} V^{-1}(x(s)) x^{T}(s) \bar{C} \sigma(r(s)) d w(s) .
\end{aligned}
$$

Consequently, one gets that

$$
0.5 \widehat{c}^{-1} \widehat{\lambda} \liminf _{t \rightarrow+\infty} \frac{1}{t} \int_{0}^{t}|x(s)| d s \geq \sum_{k=1}^{N} \pi_{k} \alpha(k) \quad \text { a.s. }
$$

which implies the other required assertion (4.12).

Similarly, using Lemmas 6, 10, and 11, we can show the following 
Theorem 13. Under Assumptions 5 and 8, for any given initial data $\{x(t):-\tau \leq t \leq 0\} \in C\left([-\tau, 0] ; R_{+}\right)$, the solution $x(t)$ of (4) obeys

$$
\begin{aligned}
& \limsup _{t \rightarrow \infty} \frac{1}{t} \int_{0}^{t}|x(s)| d s \leq \frac{1}{\lambda} \sum_{i=k}^{N} \pi_{k} \beta(k) \quad \text { a.s., } \\
& \liminf _{t \rightarrow \infty} \frac{1}{t} \int_{0}^{t}|x(s)| d s \geq \frac{2 \widehat{c}}{\widehat{\lambda}} \sum_{k=1}^{n} \pi_{k} \alpha(k) \quad \text { a.s., }
\end{aligned}
$$

where $-\hat{\lambda}=\min _{k \in S}\left[\lambda_{\min }\left(\bar{C} A(k)+A^{T}(k) \bar{C}\right)\right]<0$.

\section{Examples}

In this section, an example is given to illustrate our main results.

Example 1. Consider the two-species Lotka-Volterra system with regime switching described by

$$
\begin{aligned}
d x(t)= & \operatorname{diag}\left(x_{1}(t), x_{2}(t)\right) \\
\times[(b(r(t))+A(r(t)) x(t) & \\
& +B(r(t)) x(t-\tau)) d t+\sigma(r(t)) d w(t)],
\end{aligned}
$$

where $\left.x(t)=\left(x_{1}(t), x_{2}(t)\right)^{T}, b(r(t))=\left(b_{1} r(t)\right), b_{2}(r(t))\right)^{T}$, $\sigma(r(t))=\left(\sigma_{1}(r(t)), \sigma_{2}(r(t))\right)^{T}$,

$$
\begin{aligned}
& A(r(t))=\left(\begin{array}{ll}
a_{11}(r(t)) & a_{12}(r(t)) \\
a_{21}(r(t)) & a_{22}(r(t))
\end{array}\right), \\
& B(r(t))=\left(\begin{array}{ll}
b_{11}(r(t)) & b_{12}(r(t)) \\
b_{21}(r(t)) & b_{22}(r(t))
\end{array}\right),
\end{aligned}
$$

and $r(t)$ is a right-continuous Markov chain taking values in $S=\{1,2\}$, and $r(t)$ and $w(t)$ are independent. Here

$$
\begin{array}{lll}
b_{1}(1)=5, & a_{11}(1)=-5, & a_{12}(1)=\sqrt{10}, \\
b_{2}(1)=8, & a_{21}(1)=\sqrt{10}, & a_{22}(1)=-5, \\
b_{1}(2)=4, & a_{11}(2)=-3, & a_{12}(2)=\sqrt{2}, \\
b_{2}(2)=5, & a_{21}(2)=\sqrt{2}, & a_{22}(2)=-3, \\
b_{11}(1)=0, & b_{12}(1)=\frac{1}{2}, & \sigma_{1}(1)=\sqrt{2}, \\
b_{21}(1)=1, & b_{22}(1)=0, & \sigma_{2}(1)=2, \\
b_{11}(2)=0, & b_{12}(2)=\frac{\sqrt{2}}{2}, & \sigma_{1}(2)=\sqrt{14}, \\
b_{21}(2)=\frac{\sqrt{2}}{2}, & b_{22}(2)=0, & \sigma_{2}(2)=4 .
\end{array}
$$

Let $\bar{C}=I \in R^{2 \times 2}$. It is easy to compute that

$$
\begin{gathered}
|c|=\sqrt{2}, \quad \widehat{c}=1, \\
\max _{k \in S}\left\{\lambda_{\max }^{+}\left[\frac{1}{2}(\bar{C} A(k)+A(k) \bar{C})\right]\right\} \leq-3+\sqrt{2} \\
-\hat{\lambda}=-5-\sqrt{10}<0, \quad \max _{k \in S}\|\bar{C} B(k)\| \leq \frac{\sqrt{5}}{2} .
\end{gathered}
$$

Then

$$
\begin{aligned}
-\lambda= & |c|^{-1} \max _{k \in S}\left\{\lambda_{\max }^{+}\left[\frac{1}{2}(\bar{C} A(k)+A(k) \bar{C})\right]\right\} \\
& +\widehat{c}^{-1} \max _{k \in S}\|\bar{C} B(k)\|<0 .
\end{aligned}
$$

Moreover, $\alpha(1)=3, \alpha(2)=-4, \beta(1)=7$, and $\beta(2)=-2$.

By Theorem 1(1), the solution $x(t)$ of (85) will remain in $R_{+}$for all $t \geq-\tau$ with probability 1 . Let the generator of the Markov chain $r(t)$ be

$$
\Gamma=\left(\begin{array}{rr}
-1 & 1 \\
2 & -2
\end{array}\right)
$$

By solving the linear equation $\pi \Gamma=0$, we obtain the unique stationary (probability) distribution $\pi=\left(\pi_{1}, \pi_{2}\right)=$ $(2 / 3,1 / 3)$. Then

$$
\sum_{1=1}^{2} \pi_{k} \alpha(k)=\frac{2}{3}>0, \quad \sum_{1=1}^{2} \pi_{k} \beta(k)=4>0 .
$$

Therefore, by Theorems 8 and 12, (85) is stochastically permanent and the solutions have the following properties:

$$
\begin{aligned}
\frac{2}{\widehat{\lambda}} & \leq \liminf _{t \rightarrow \infty} \frac{1}{t} \int_{0}^{t}|x(s)| d s \\
& \leq \limsup _{t \rightarrow \infty} \frac{1}{t} \int_{0}^{t}|x(s)| d s \leq \frac{4}{\lambda} \quad \text { a.s. }
\end{aligned}
$$

\section{Acknowledgments}

The authors are grateful to Editor Proffessor Yuming Chen for the diligent work. This work is supported by Research Fund for Doctor Station of Ministry of Education of China (no. 20113401110001, no. 20103401120002), TIAN YUAN Series of Natural Science Foundation of China (no. 11126177, no. 11226247), Key Natural Science Foundation (no. KJ2009A49), 211 Project of Anhui University (no. KJJQ1101), Anhui Provincial Nature Science Foundation (no. 1308085MA01, no. 1308085QA15, and no. 1208085QA15), and Foundation for Young Talents in College of Anhui Province (no. 2012SQRL021).

\section{References}

[1] S. Ahmad and M. R. M. Rao, "Asymptotically periodic solutions of $n$-competing species problem with time delays," Journal of Mathematical Analysis and Applications, vol. 186, no. 2, pp. 559571,1994 
[2] H. Bereketoglu and I. Győri, "Global asymptotic stability in a nonautonomous Lotka-Volterra type system with infinite delay," Journal of Mathematical Analysis and Applications, vol. 210, no. 1, pp. 279-291, 1997.

[3] H. I. Freedman and S. G. Ruan, "Uniform persistence in functional-differential equations," Journal of Differential Equations, vol. 115, no. 1, pp. 173-192, 1995.

[4] K. Gopalsamy, Stability and Oscillations in Delay Differential Equations of Population Dynamics, Kluwer Academic Publishers, Dordrecht, The Netherlands, 1992.

[5] V. Kolmanovskiı̌ and A. Myshkis, Applied Theory of FunctionalDifferential Equations, Kluwer Academic Publishers, Dordrecht, The Netherlands, 1992.

[6] Y. Kuang, Delay Differential Equations with Applications in Population Dynamics, Academic Press, Boston, Mass, USA, 1993.

[7] A. Bahar and X. Mao, "Stochastic delay population dynamics," International Journal of Pure and Applied Mathematics, vol. 11, no. 4, pp. 377-400, 2004.

[8] X. Mao, S. Sabanis, and E. Renshaw, "Asymptotic behaviour of the stochastic Lotka-Volterra model," Journal of Mathematical Analysis and Applications, vol. 287, no. 1, pp. 141-156, 2003.

[9] X. Mao, G. Marion, and E. Renshaw, "Environmental Brownian noise suppresses explosions in population dynamics," Stochastic Processes and their Applications, vol. 97, no. 1, pp. 95-110, 2002.

[10] D. Jiang and N. Shi, "A note on nonautonomous logistic equation with random perturbation," Journal of Mathematical Analysis and Applications, vol. 303, no. 1, pp. 164-172, 2005.

[11] D. Jiang, N. Shi, and X. Li, "Global stability and stochastic permanence of a non-autonomous logistic equation with random perturbation," Journal of Mathematical Analysis and Applications, vol. 340, no. 1, pp. 588-597, 2008.

[12] T. C. Gard, Introduction to Stochastic Dierential Equations, Marcel Dekker, 1998.

[13] A. Bahar and X. Mao, "Stochastic delay Lotka-Volterra model," Journal of Mathematical Analysis and Applications, vol. 292, no. 2, pp. 364-380, 2004.

[14] X. Mao, "Delay population dynamics and environmental noise," Stochastics and Dynamics, vol. 5, no. 2, pp. 149-162, 2005.

[15] S. Pang, F. Deng, and X. Mao, "Asymptotic properties of stochastic population dynamics," Dynamics of Continuous, Discrete \& Impulsive Systems A, vol. 15, no. 5, pp. 603-620, 2008.

[16] H. Huang, Z. Wu, and L. Wang, " $\psi^{\gamma}$ stability analysis for neutral stochastic neural networks with multiple delays based on LMI approach," Journal of Biomathmatics. In press.

[17] Z. Wu, H. Huang, and L. Wang, "Dynamical behavior of a stochastic ratio-dependent predator-prey system," Journal of Applied Mathematics, vol. 2012, Article ID 857134, 17 pages, 2012.

[18] Z. Wu, H. Huang, and L. Wang, "Exponential stability of impulsive stochastic functional differential systems," Abstract and Applied Analysis, vol. 2012, Article ID 678536, 12 pages, 2012.

[19] Z. Wu, H. Huang, and L. Wang, "Stochastic delay logistic model with markovian switching," International Journal of Information and Systems Science, vol. 8, no. 2, pp. 174-180, 2012.

[20] Z. Wu, H. Huang, and L. Wang, "Stochastic delay logistic model under regime switching," Abstract and Applied Analysis, vol. 2012, Article ID 241702, 26 pages, 2012.

[21] Y. Takeuchi, N. H. Du, N. T. Hieu, and K. Sato, "Evolution of predator-prey systems described by a Lotka-Volterra equation under random environment," Journal of Mathematical Analysis and Applications, vol. 323, no. 2, pp. 938-957, 2006.

[22] Q. Luo and X. Mao, "Stochastic population dynamics under regime switching," Journal of Mathematical Analysis and Applications, vol. 334, no. 1, pp. 69-84, 2007.

[23] X. Li, D. Jiang, and X. Mao, "Population dynamical behavior of Lotka-Volterra system under regime switching," Journal of Computational and Applied Mathematics, vol. 232, no. 2, pp. 427-448, 2009.

[24] X. Li, A. Gray, D. Jiang, and X. Mao, "Sufficient and necessary conditions of stochastic permanence and extinction for stochastic logistic populations under regime switching," Journal of Mathematical Analysis and Applications, vol. 376, no. 1, pp. 1128, 2011.

[25] C. Zhu and G. Yin, "On hybrid competitive Lotka-Volterra ecosystems," Nonlinear Analysis. Theory, Methods \& Applications A, vol. 71, no. 12, pp. el370-e1379, 2009.

[26] M. Liu and K. Wang, "Persistence and extinction of a stochastic single-specie model under regime switching in a polluted environment," Journal of Theoretical Biology, vol. 264, no. 3, pp. 934-944, 2010.

[27] M. Liu and K. Wang, "Asymptotic properties and simulations of a stochastic logistic model under regime switching," Mathematical and Computer Modelling, vol. 54, no. 9-10, pp. 2139-2154, 2011.

[28] Z. Wu, H. Huang, and L. Wang, "Stochastic delay population Dynamics under regime switching: global solutions and extinction," Abstract and Applied Analysis, vol. 2013, Article ID 918569, 10 pages, 2013.

[29] X. Mao and C. Yuan, Stochastic Differential Equations with Markovian Switching, Imperial College Press, London, UK, 2006. 


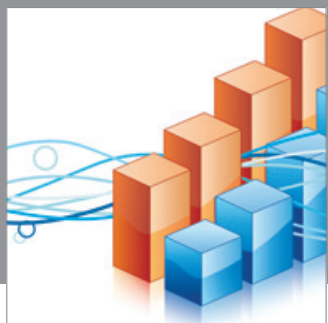

Advances in

Operations Research

mansans

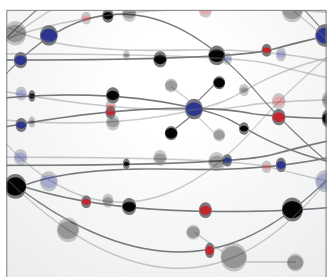

The Scientific World Journal
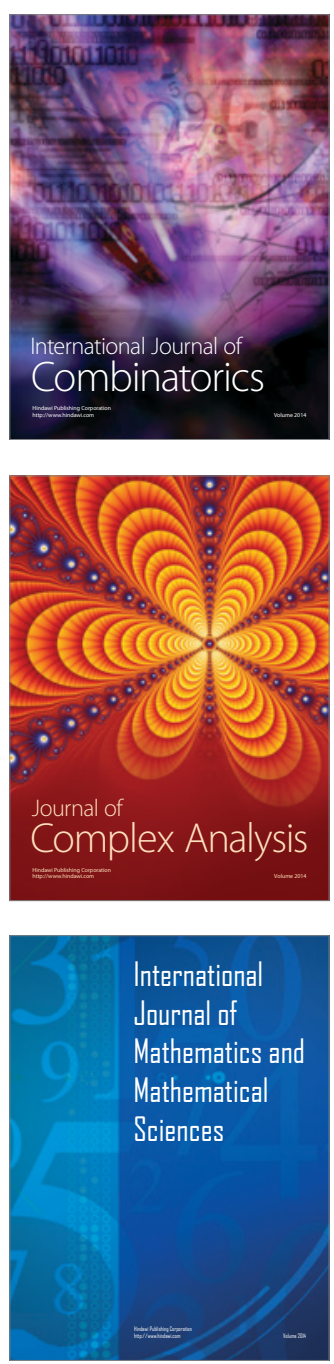
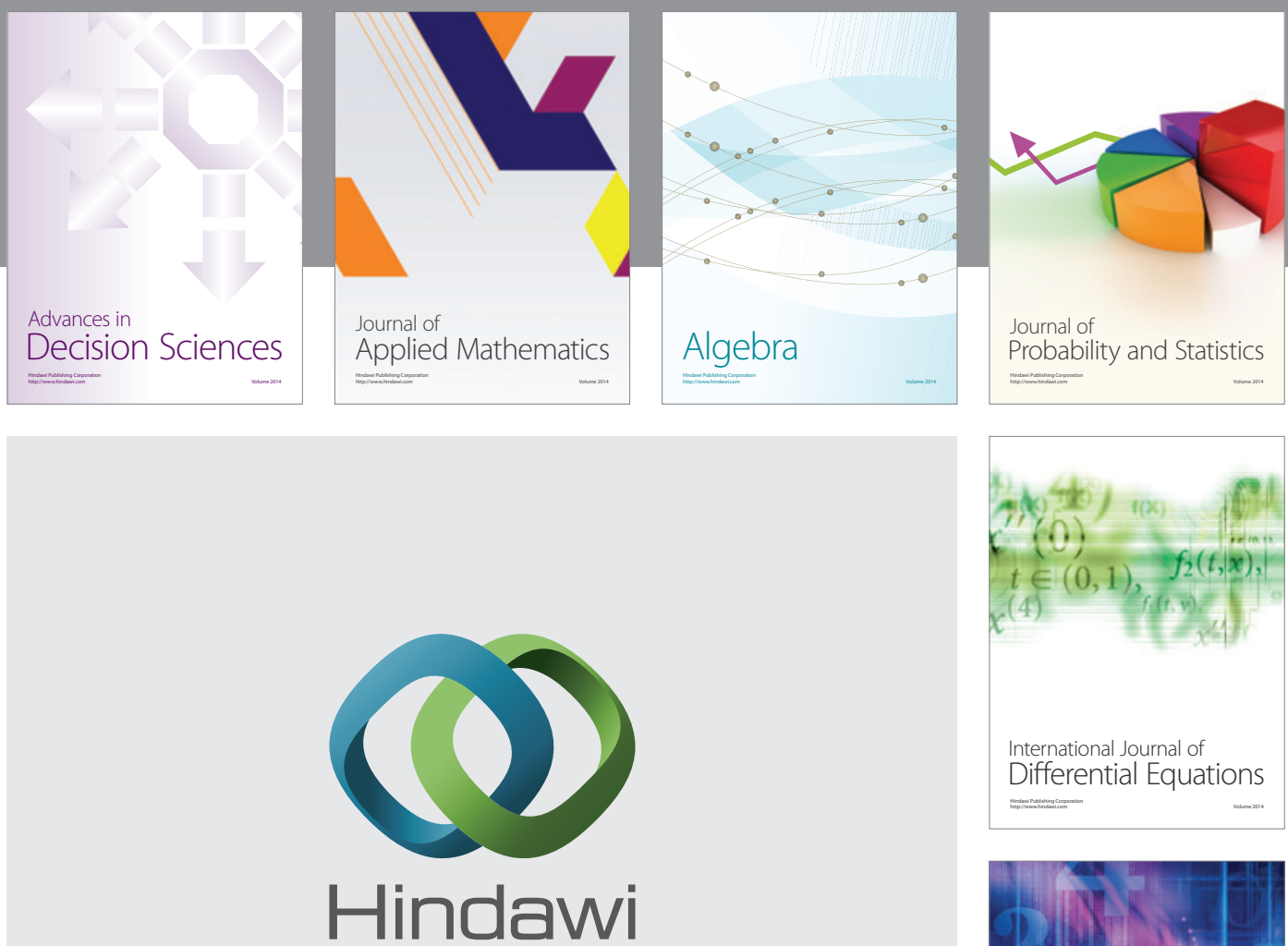

Submit your manuscripts at http://www.hindawi.com
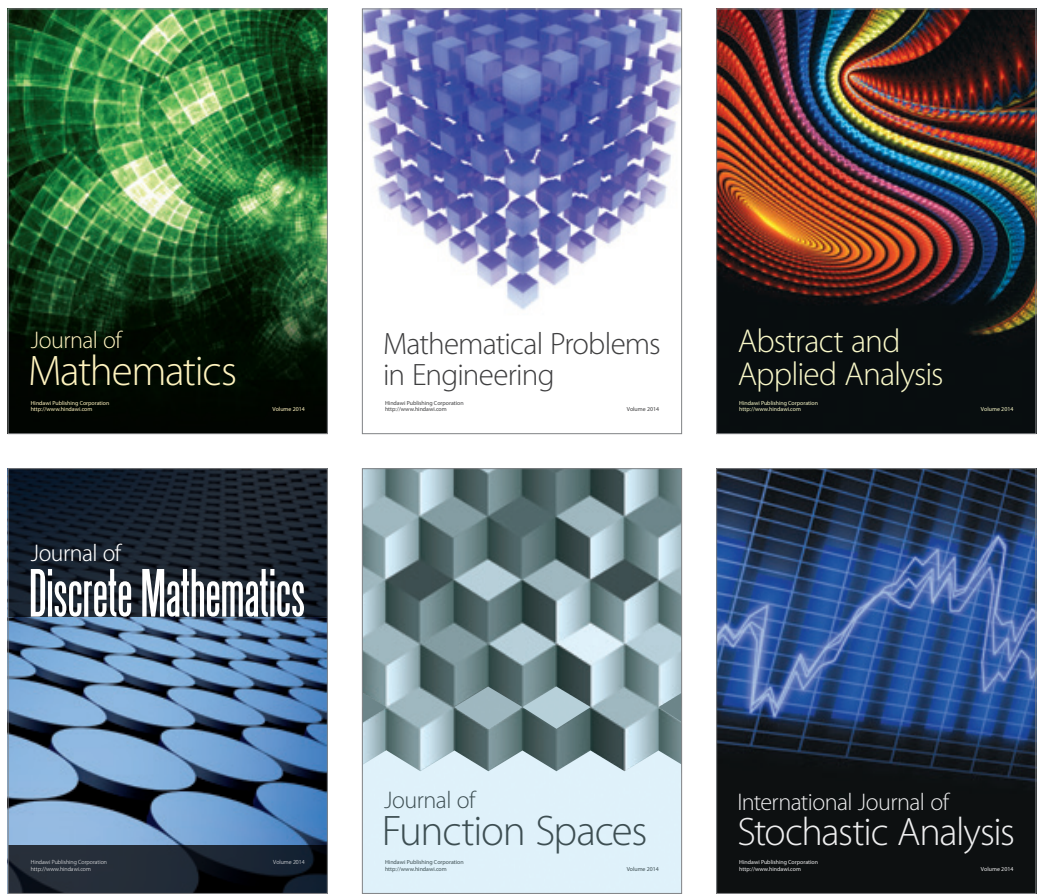

Journal of

Function Spaces

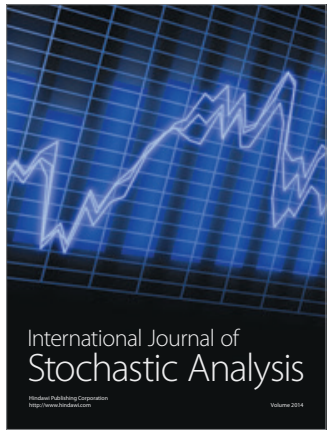

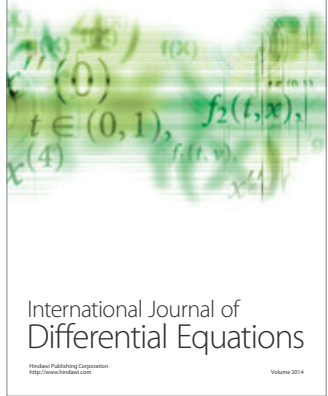
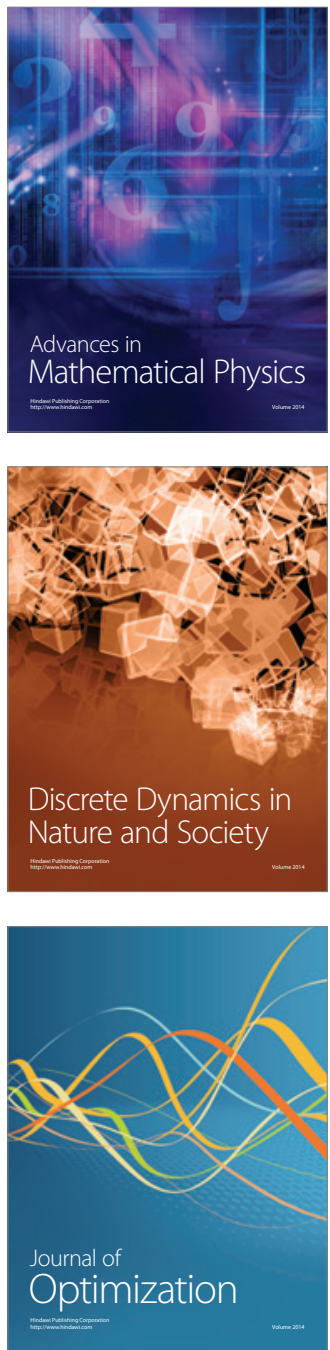